\title{
Exponential Gravity
}

\author{
Eric V. Linder \\ Berkeley Lab \& University of California, Berkeley, CA 94720, USA \\ Institute for the Early Universe, Ewha Womans University, Seoul, South Korea
}

(Dated: October 26, 2018)

\begin{abstract}
We investigate a $f(R)$ modification of gravity that is exponential in the Ricci scalar $R$ to explain cosmic acceleration. The steepness of this dependence provides extra freedom to satisfy solar system and other curvature regime constraints. With a parameter to alleviate the usual fine tuning of having the modification strengthen near the present, the total number of parameters is only one more than $\Lambda \mathrm{CDM}$. The resulting class of solutions asymptotes to $w=-1$ but has no cosmological constant. We calculate the dynamics in detail, examine the effect on the matter power spectrum, and provide a simple fitting form relating the two.
\end{abstract}

\section{INTRODUCTION}

Einstein's general relativity has proved to be a remarkably robust theory of gravity. Large numbers of attempts have been made to modify it, e.g. for the purpose of explaining cosmic acceleration without adding a separate dark energy component. Many of these have been found to have pathologies such as ghosts, unbounded energies, tachyons, or other ills [1]. Gravity theories that are sound may still fail to provide acceleration, an early matter dominated period, or fall afoul of gravity constraints on solar system or other scales.

Here we work within the framework of $f(R)$ theories that generalize the linear dependence on the Ricci scalar $R$ in the Einstein-Hilbert action. Many such viable models exist but tend to have a restricted range obeying structure constraints, and in this regime are effectively identical to $\Lambda \mathrm{CDM}$ as far as the expansion history and distances. Furthermore these often take the characteristic curvature scale for the modification to the action to be that of the present matter density, i.e. when $R=8 \pi G \rho_{\text {today }}$, so that the modifications kick in near today.

We explore ways of ameliorating all three issues: easing the restrictions of solar system tests and structure constraints on the curvature evolution, loosening the expansion history, and relaxing the fine tuning.

Considerable work has been done on $f(R)$ theories; see [2, 3] for overall reviews and references. In this paper we follow most closely the setup of [4, [5, [6]. Our aim is to investigate the dynamics in more detail than usual, and relate it to the growth effects more explicitly, keeping close touch with the cosmological observations of both. In $₫$ II we describe the model, its equations of motion, and the solutions for the expansion history and effective equation of state. The effects on systems with high curvature and density gradients, such as the solar system and galaxies, are discussed in $₫$ III along with the growth of structure.

\section{MODEL AND DYNAMICS}

The $f(R)$ class of extensions to general relativity represents a simple modification that preserves the maximum number of derivatives entering at two, and can have a well defined limit in which the theory reduces to general relativity, typically in the high scalar curvature regime. The gravitational part of the modified Einstein-Hilbert action is

$$
S=\frac{1}{16 \pi G} \int d^{4} x \sqrt{-g}[R+f(R)],
$$

where $g$ is the determinant of the metric and $R$ is the Ricci scalar curvature.

In order to avoid including an implicit cosmological constant we require the low curvature limit of $f$ to vanish, i.e. $f(0)=0$. At high curvature (e.g. in the early universe), we want the modification term to be negligible compared to the usual term linear in $R$, so we require $f(R \gg 1) / R \rightarrow 0$. We do not want to put in by hand a specific preference for dynamics at the present, i.e. a fine tuning, so we do not set the characteristic scale of $f$ in terms of $R_{0}=8 \pi G \rho(a=1)$, where $a$ is the cosmic scale factor and $\rho$ the matter density. Rather, we give $f$ a transition scale $r$ and will fit for $r$ given observations. Finally, in order to satisfy the constraints from locally high curvature systems such as the solar system or galaxies that gravity must be very close to general relativity, we take $f$ to have a steep dependence on scalar curvature, rapidly restoring Einstein gravity.

This steepness will be a key ingredient in improving agreement with structure constraints while ameliorating fine tuning. Steep potentials have been considered previously (see, e.g., [5, 7, [8, 9, 10]) but here we explore in substantial detail the dynamics, from the deviation from matter domination, to the maximum equation of state variation, to the long time behavior in the equation of state function $w(a)$ and phase space $w-w^{\prime}$. We also give a quantitative analysis of effects on the matter power spectrum, as well as an accurate fitting form relating the expansion and growth effects. These results are of interest apart from the specific form adopted. We now seek the simplest form with fewest parameters yet freedom to 
fit over a variety of characteristic curvature scales, not putting in special behavior just at the present density.

All these conditions are satisfied by the ansatz

$$
f(R)=-\operatorname{cr}\left(1-e^{-R / r}\right) .
$$

This simple form involves two quantities, and in fact we will see that the combination $c r$ is equivalent to setting the dimensionless matter density today $\Omega_{m}$, so there is only one parameter besides $\Omega_{m}$. Thus for this form the complexity is just one step more than the standard $\Lambda \mathrm{CDM}$ model. We focus on cosmological gravity from the matter domination through the accelerating eras, i.e. from high curvature to the asymptotic future; note primordial nucleosynthesis will not be altered, as discussed in Sec. IIII

The Friedmann equation of motion is modified to [4]

$$
\begin{aligned}
H^{2}+\frac{f}{6} & -f_{R}\left[\frac{1}{2}\left(H^{2}\right)^{\prime}+H^{2}\right]+H^{2} f_{R R} R^{\prime} \\
& =\frac{8 \pi G}{3} \rho(a) \equiv m^{2} a^{-3}
\end{aligned}
$$

where a prime denotes $d / d \ln a$, a subscript $R$ denotes a derivative with respect to $R, H$ is the Hubble parameter, and $\rho$ is the matter density, taken to be the only physical component of energy density. Note $R=12 H^{2}+3\left(H^{2}\right)^{\prime}$, and for our exponential gravity model $f_{R R}=(c / r) e^{-R / r}$. Thus $f_{R R}>0$, a critical stability condition (see [2] for a review).

Following [5] with some modifications we define

$$
\begin{aligned}
& x_{H}=\frac{H^{2}}{m^{2}}-a^{-3}-\frac{c}{6} \frac{r}{m^{2}}, \\
& x_{R}=\frac{R}{m^{2}}-3 a^{-3}-2 c \frac{r}{m^{2}}-12 x_{H},
\end{aligned}
$$

to take out the leading order terms. The equation of motion (3) becomes two coupled first order equations

$$
\begin{aligned}
x_{H}^{\prime} & =x_{R} / 3, \\
x_{R}^{\prime} & =-4 x_{R}-\frac{1}{m^{2} f_{R R}} \frac{x_{H}+\left[d-9\left(\mathrm{~cm}^{2} / r\right) a^{-6}\right] e^{-R / r}}{a^{-3}+x_{H}+d} \\
& +9 \frac{x_{H}+d}{1+a^{3}\left(x_{H}+d\right)}+\frac{f_{R}}{m^{2} f_{R R}}\left(\frac{R}{6 H^{2}}-1\right),
\end{aligned}
$$

where $d=c r /\left(6 m^{2}\right)$ and

$$
\frac{R}{6 H^{2}}=\frac{1}{2} \frac{1+a^{3}\left(4 d+4 x_{H}+x_{R} / 3\right)}{1+a^{3}\left(x_{H}+d\right)} .
$$

In this form we have carried out the explicit cancellation of several terms that nominally appeared dominant, making the numerical solution more robust.

Treating the terms modifying the Friedmann equation (3) as an effective dark energy density $\Omega_{d e}(a)$, we can define the effective dark energy equation of state and its variation,

$$
\begin{aligned}
\Omega_{d e}(a) & =\frac{x_{H}+d}{a^{-3}+x_{H}+d}, \\
w & =-1-\frac{1}{9} \frac{x_{R}}{x_{H}+d}, \\
w^{\prime} & =3(1+w)^{2}+(1+w) \frac{x_{R}^{\prime}}{x_{R}} .
\end{aligned}
$$

In the future, we see that $\Omega_{d e} \rightarrow 1$, i.e. the modifications dominate and the effective dark energy density goes to the critical density. In the high curvature regime, $f$ goes to a constant, $-c r$, and so one expects this to be related to $\Omega_{\Lambda}$, or $1-\Omega_{m}$ in the spatially flat universe we consider. In general, the exact expression for $\mathrm{cr}$ is given by

$$
d \equiv \frac{c}{6} \frac{r}{m^{2}}=\Omega_{m}^{-1}-1-x_{H},
$$

so there is a defined relation between $\mathrm{cr}$ and $\Omega_{m}$. When the curvature is large, $x_{H} \sim e^{-R / r}$ (see below) and can be neglected and the product $c r$ is explicit in terms of $\Omega_{m}$. Since $c r$ corresponds to $\Omega_{m}$ (all plots are for $\Omega_{m}=0.28$ ), this leaves $c$ as the only other parameter of the theory.

The dynamics can also be understood fairly simply. In the high curvature limit when both $x_{H}$ and $x_{R}$ are small, $w \rightarrow-1$, i.e. it starts from a frozen, cosmological constant state during the matter dominated expansion. Expanding the equations of motion at high redshift one finds that $x_{H}, x_{R} \sim e^{-R / r}$ and are positive. So from Eq. (10) one sees that the field starts off phantom, i.e. with $w<-1$, and since $x_{R}^{\prime} / x_{R} \sim a^{-3}$ then from Eq. (11) the field evolves quite rapidly, $w^{\prime} \sim a^{-3}(1+w)$, much faster than a physical thawing scalar field which has $w^{\prime}=$ $3(1+w)[11,12]$.

Solving the equations of motion numerically, we display the effective phase space dynamics of $w^{\prime}-w$ in Fig. 1. We indeed see evolution from a frozen, cosmological constant state $\left(w=-1, w^{\prime}=0\right)$ to the phantom regime, more swiftly than thawing ("sublimation"), and then an oscillatory behavior where the field reaches a maximum value of $|1+w|$, then crosses back over $w=-1$, reaches a secondary maximum and quickly damps around $w=-1$. Note the second period of oscillations is highly damped, so small on the figure it appears as just a short dash around $w=-1$.

In fact, there is an asymptotic behavior toward an effective cosmological constant. The stationary point of the equation of motion is given by

$$
\begin{aligned}
& x_{R}=0, \\
& x_{H}=-d(c+1)\left(e^{R / r}+c\right)^{-1},
\end{aligned}
$$

with an implicit relation for $R$ of

$$
\frac{R}{m^{2}}=12 d \frac{e^{R / r}-1}{e^{R / r}+c}
$$

So $R$ settles asymptotically to a constant value, as does $H$, meaning that the future solution possesses $w=-1$. 


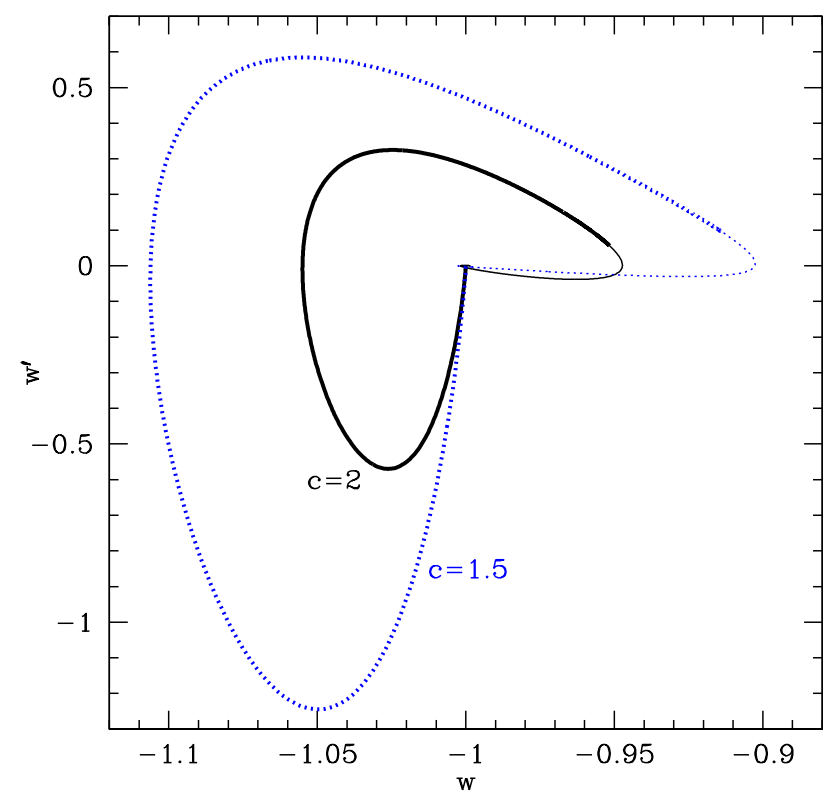

FIG. 1: The phase space dynamics of the effective dark energy parameters $w^{\prime}-w$ is unlike any standard scalar field. It crosses between the phantom and normal parts of the phase space (often a signal of modified gravity), and "sublimates" rather than thaws from the initial frozen state. Successive oscillations about $\Lambda$ are barely visible here, showing rapid damping to the asymptotic cosmological constant. Thick portions of the curves show the evolution for $a \leq 1$.

When the asymptotic value of $e^{R / r}$ is much greater than one then $R / m^{2} \rightarrow 12 d$, or 30.86 for $\Omega_{m}=0.28$. No valid attractor solution exists for $c<1$, because this would require $R<0$.

Figure 2 illustrates the numerical solution for $R(a)$ for several values of $c$. We see that as promised $R$ evolves very steeply due to the exponential form of $f(R)$ and is in the high curvature limit $e^{R / r} \gg 1$ until recently. It then quickly reaches its asymptotic value given by Eq. (15). As $c$ increases, $r$ necessarily decreases to preserve $c r$, i.e. $\Omega_{m}$, and so $e^{R / r}$ is always large and asymptotically $R / m^{2}=$ 30.86. Thus, the evolution of $R(a)$ is quite similar for all $c \gtrsim 3\left(r / m^{2} \lesssim 5\right)$. The influence of the modification rises near the present for any allowed values of $r$, without additional fine tuning.

To examine the expansion history in more detail, we consider the effective dark energy equation of state $w(a)$ induced by the gravity modifications. Figure 3 shows this function for a variety of different values of $c$. As discussed, the equation of state goes from $w=-1$ at high redshift to $w<-1$ then crosses back to $w>-1$. The maximum departure from $w=-1$ decreases rapidly as $c$ increases, going roughly as $e^{-2 c}$ for large $c$. Thus as $c$ gets very large, any deviations from $\Lambda \mathrm{CDM}$ are strongly suppressed. Figure 4 extends the plot to the future, showing

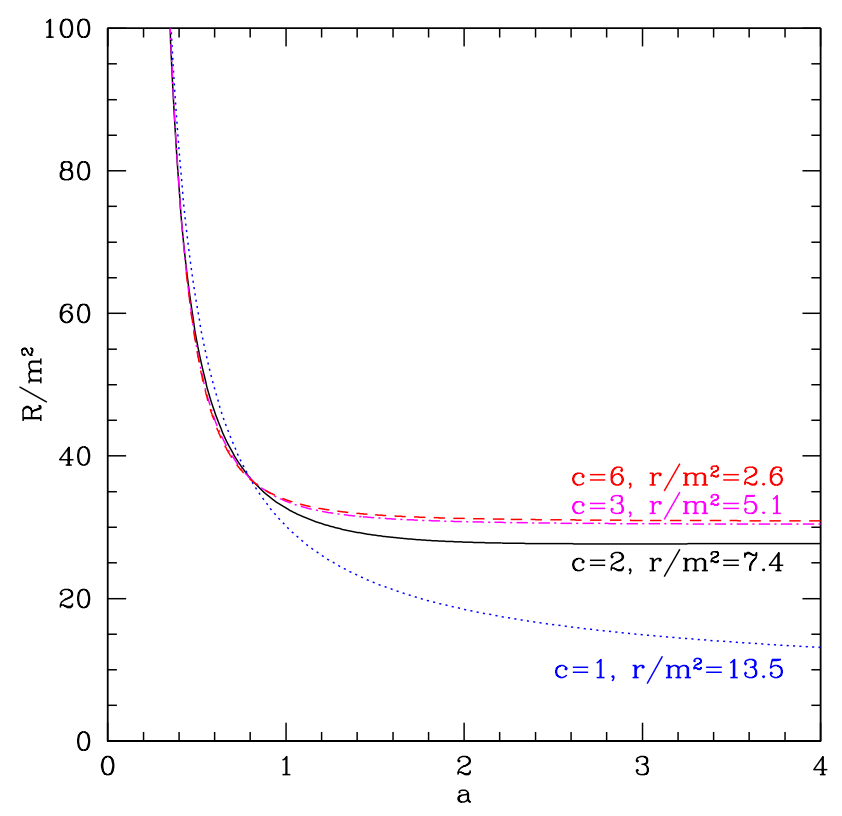

FIG. 2: The Ricci scalar curvature, normalized by the present matter density, evolves very rapidly from the high redshift, high curvature state to a constant asymptotic state (for $c>$ 1 ). As $c$ gets larger than unity, $R / m^{2}$ approaches a constant given by $12\left(\Omega_{m}^{-1}-1\right)$ (i.e. 30.86 for $\Omega_{m}=0.28$ as here) and the modification factor $e^{R / r}$ always stays large. The influence of the modification occurs near the present for any allowed values of $r$, without additional fine tuning.

the rapidly damped oscillation around $w=-1$.

From the perspective of expansion history measurements, the distance to the cosmic microwave background last scattering surface agrees with the $\Lambda \mathrm{CDM}$ model with the same present matter density to $0.54 \%(0.2 \%)$ for all valid $c$ (for $c>1.5$ ). Distances to redshifts $z<2$, e.g. as measured by the Type Ia supernovae magnitude-redshift relation, agree to $2.3 \%(1 \%)$ for all valid $c$ (for $c>1.6$ ). The parenthetical values for each correspond roughly to next generation observational limits. However, we will see in the next section that cosmic structure and its growth impose more severe limits on the parameter values allowed.

\section{CURVATURE, STRUCTURE, AND GROWTH}

The $f(R)$ class of models of most interest acts like general relativity at high curvatures, so as to preserve agreements with primordial nucleosynthesis and other early time physics. However they possess additional scalar degrees of freedom that can become apparent in lower curvature or high density gradient regimes [5]. Treating $f_{R}$ as a scalar field one can define a Compton wavelength below which the effects on structure formation 


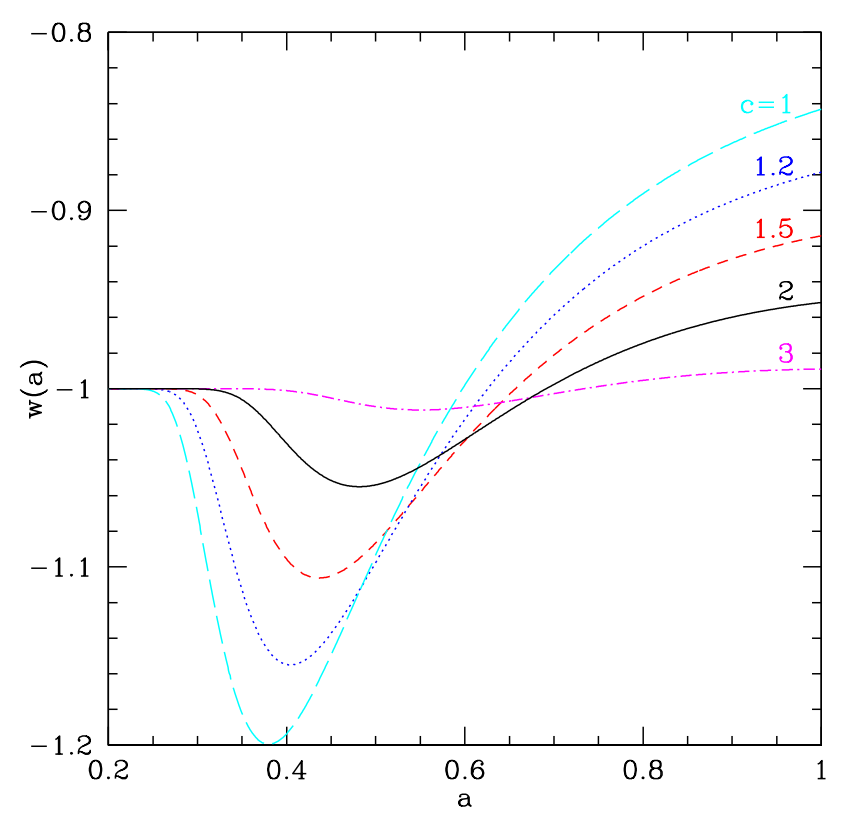

FIG. 3: The effective dark energy equation of state evolution is shown for various values of $c$. As $c$ gets large, the expansion history becomes indistinguishable from $\Lambda \mathrm{CDM}$.

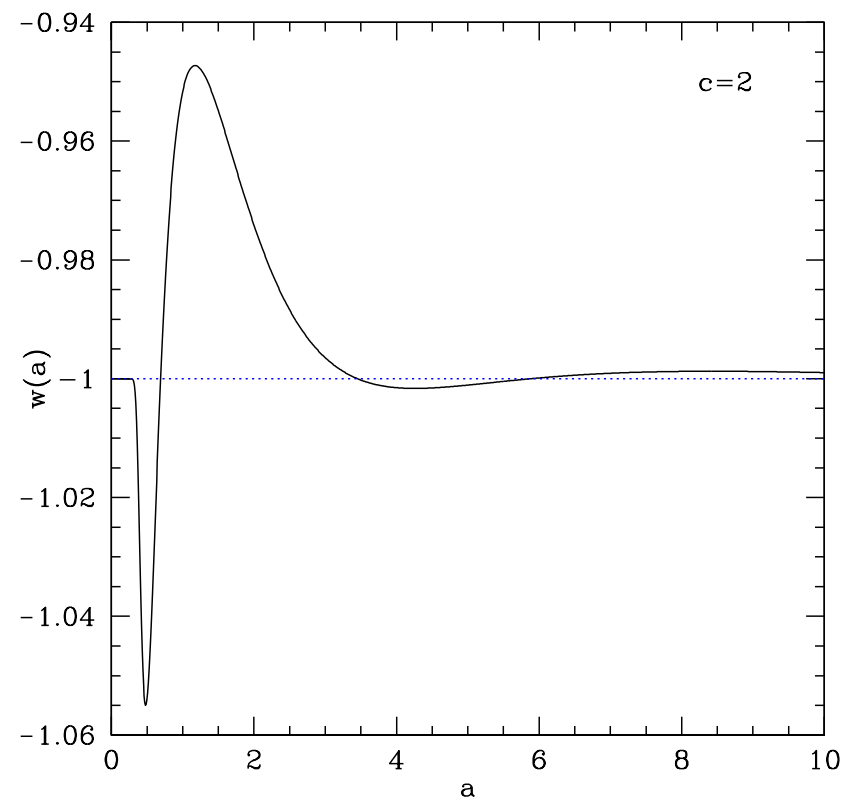

FIG. 4: The long term history of the effective dark energy equation of state is evolution from a cosmological constant state $(w=-1)$, deviation to both the phantom $(w<-1)$ and normal $(w>-1)$ sides, and rapid damping to a future cosmological constant state.

and bound structures become significantly different from general relativity. For example, the first parametrized post-Newtonian parameter, $\gamma$, takes the value $1 / 2$ rather than unity as in general relativity. (In the high curvature regime inside the structure, general relativity is restored by the chameleon mechanism [13], under conditions detailed by, e.g., [6], which the exponential model can satisfy. We address this further below.)

The key indicators to these effects are the quantities $f_{R}$ and the effective Compton wavelength with respect to $f_{R}$, usually written in terms of $[4,[5]$

$$
B=\frac{f_{R R}}{1+f_{R}} R^{\prime} \frac{2 H^{2}}{\left(H^{2}\right)^{\prime}},
$$

where $B$ is the square of the wavelength in units of the Hubble scale. Note that $1+f_{R}>0$ in the exponential model for all $c$ giving a finite attractor solution, i.e. $c>1$. This even holds during the radiation era since although we are used to thinking that $R=0$ then, the value is in fact $R \ll \rho_{\text {rad }}-$ but one still has $R \sim 8 \pi G \rho_{m, 0} a^{-3} \gg$ $8 \pi G \rho_{m, 0} \sim r$ so $R / r \gg 1$ at least back through primordial nucleosynthesis and so $1+f_{R} \rightarrow 1$. The condition $B>0$, together with the vanishing of derivatives of $f$ at high redshift, delivers a standard radiation era [4]. Given the scale $B$, modifications to structure occur for wavenumbers $k>a H / B^{1 / 2}$. This will begin to affect linear perturbation growth, $k<0.1 h / \mathrm{Mpc}$ say, for $B \gtrsim 10^{-5}$. We will later calculate a more exact observational constraint.

The exponential form of Eq. (2) ameliorates the issues involved with deviations from general relativity in structure constraints, since $f_{R}$ and $B \sim e^{-R / r}$. First, the evolution of the curvature and its effects are much more rapid than in many $f(R)$ theories, so for a given present value of curvature, or $f_{R}$, the deviation from general relativity can be much smaller in the past, when the structure formed, at $a=0.5$ say. To rigorously quantify this argument requires numerical simulations of nonlinear structure formation in the specific model of modified gravity to robustly compute the "leaking" of the nongeneral relativity influence into the structure over time, as in [14]. If we can take advantage of this increased latitude, we can allow the characteristic curvature scale $r$ to be more relaxed from the present matter density, giving greater freedom in the model.

Figure 5 shows the steep dependence of $f_{R}$ and $B$ with scale factor $a$ and how this allows a range for the characteristic scale $r$, or equivalently the parameter $c$ (related by Eq. [12). For example, in the $n=4$ model of [5] the value of $B$ drops by a factor 3 from the present to $a=0.5$ (redshift $z=1$ ), while the exponential model achieves a drop of $300(30,000)$ for $c=5(c=8)$. While the characteristic scale often seen in the literature is $m^{2}$, e.g. $f(R)$ varies as $\left(R / m^{2}\right)^{n}$, here the dependence $e^{-R / r}$ permits $r$ to lie in the range $r / m^{2}<3.8$ and still satisfy $f_{R}(a=0.5)<10^{-6}$. Since $m^{2}$ is determined by the matter density today, this freedom somewhat eases the coincidence between the characteristic scale and today's curvature. 
This steep dependence also somewhat eases the condition for bound structures to recover general relativistic behavior: while the solar system, lying in the high curvature background of the galaxy, satisfies the conditions easily, our galaxy halo requires values of $f_{R} \lesssim 10^{-6}$ (see [5], Sec. IIID for a detailed calculation). The sharp drop in $f_{R}$ when going to higher redshifts, as mentioned above, is stronger for the exponential model than most $f(R)$ theories. Still, galaxy constraints will play a significant role and we should be cautious of values of $c<4$ (imposing the condition at $a=0.5$, or $c<7$ if at $a=1$ ) failing to live up to the chameleon mechanism.

In $f(R)$ models of modified gravity it is extremely difficult to get an appreciable deviation from $\Lambda$ CDM behavior on the expansion side of observations while still obeying constraints from the structure side. Using the very steep dependence of the modifications on curvature, and hence a rapid redshift dependence of the key structure parameter $f_{R}$ (or $B$ ), exponential gravity can again do better than most models to ameliorate the situation. If we were to impose $f_{R}<10^{-6}$, say, from structure constraints, this would imply a maximum deviation in the equation of state (occurring at the peak of the first phantom excursion) $|1+w|_{\max }<4 \times 10^{-6}$ and $c>7$ if evaluated at the present, but $|1+w|_{\max }<2 \times 10^{-3}$ and $c>4$ if evaluated at the time the structure forms, say $a=0.5$. So even pushing all values as far as allowed, it is almost impossible to get an appreciable signal in $1+w$. (Note that assuming the characteristic curvature modification scale is defined by the present matter density, i.e. $r / m^{2}=1$, gives $c=15.4$.) Interestingly, we find $|1+w|_{\max }$ is excellently approximated by $(1 / 2) B(a=1)$, relating the expansion and structure sides.

We can check the magnitude of deviations from $\Lambda \mathrm{CDM}$ growth of structure, at least in the linear regime, by solving for the evolution of linear density perturbations. We take into account both the change in the gravitational coupling strength (i.e. Newton's constant) and the scaledependent effect of anisotropic stress in the $f(R)$ model (see, e.g., 7, 15]). Figure[6]shows the fractional deviation in the matter power spectrum as a function of wavenumber today.

The deviations become noticeable on the $f_{R}$ Compton scale and have a magnitude determined by $B$. Due to the steepness of the exponential model, the deviations are not as severe for a given $f_{R}(a=1)$ as for shallower $f(R)$ models. In addition, the enhanced freedom in the characteristic scale allows models to be viable for values of $c$ much lower than the $c=15.4$ given by $r / m^{2}=1$. For $c>5$, the power spectrum deviations are less than $5 \%$ at $k=0.1 \mathrm{~h} / \mathrm{Mpc}$, beyond which linear treatment must give way to numerical simulations.

Interestingly, the steepness also quickly reduces the deviations in the growth as one goes to higher redshift. The $c=3$ model with the largest deviations shown ( $12 \%$ at the extreme), has less than $1 \%$ deviations in the power spectrum at $a=0.5$. Recall that $B$ can drop by two or three orders of magnitude in this model between $a=1$

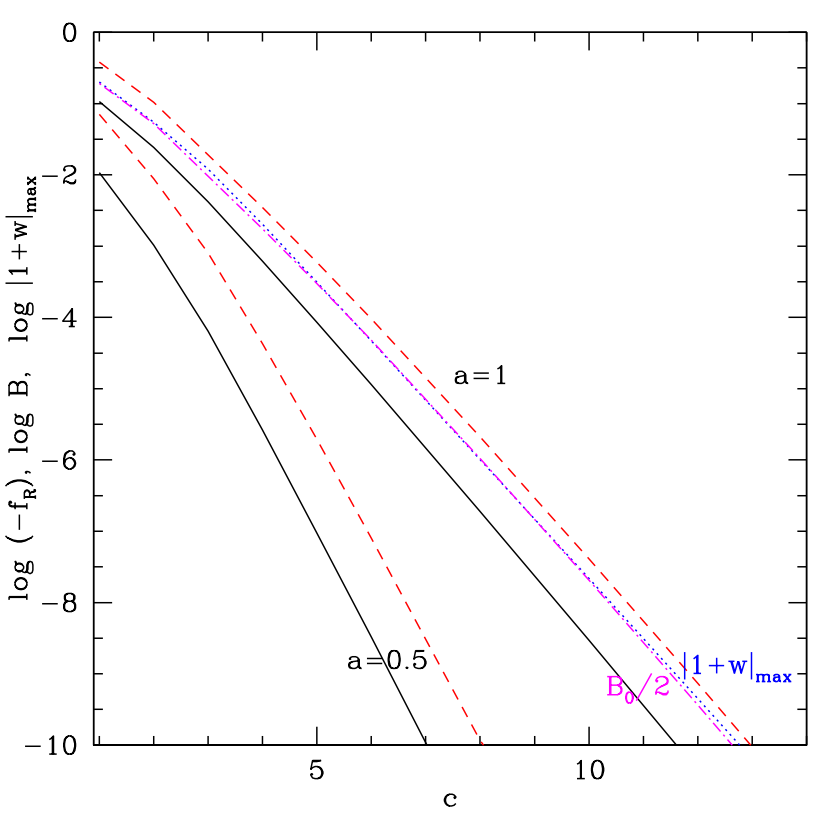

FIG. 5: The derivative $f_{R}$ (solid black curves) and the structure parameter $B$ (dashed red), related to the Compton wavelength of the scalar part of the gravity modification, are plotted vs. $c$. The upper pair is evaluated at the present and the lower pair is at $a=0.5$. Note that $f_{R}$ (and $B$ ) has a very steep dependence on scale factor $a$, so structures that might nominally have $f_{R} \approx 4 \times 10^{-4}$ today may have formed when $f_{R}<10^{-6}$ and so not exhibit observable deviations from general relativity. The maximum deviation of the effective equation of state from a cosmological constant (dotted blue) closely follows $B(a=1) / 2$ (dot-dash magenta).

and $a=0.5$. Note that because the expansion histories of $c>3$ models are so close to $\Lambda \mathrm{CDM}$, one can interpret Fig. 6 also as showing the deviation in growth from a general relativity model with the same expansion history as the $f(R)$ models. We have verified this directly by turning off the gravity modifications in the source term of the perturbation equation: the maximum change is below $0.2 \%$. Thus this figure shows the effects of modifying gravity for matched expansion.

\section{CONCLUSIONS}

The idea of explaining cosmic acceleration without adding a separate energy density component is attractive. Einstein's general relativity has proved to be highly resistant to modification, however, and in excellent accord with observations. Theories generalizing the linear factor of the Ricci scalar curvature to a more general function, $f(R)$ theories, are one of the main survivors for modifying gravity.

In this paper we explored a simple model exponential in the curvature. This has several beneficial conse- 


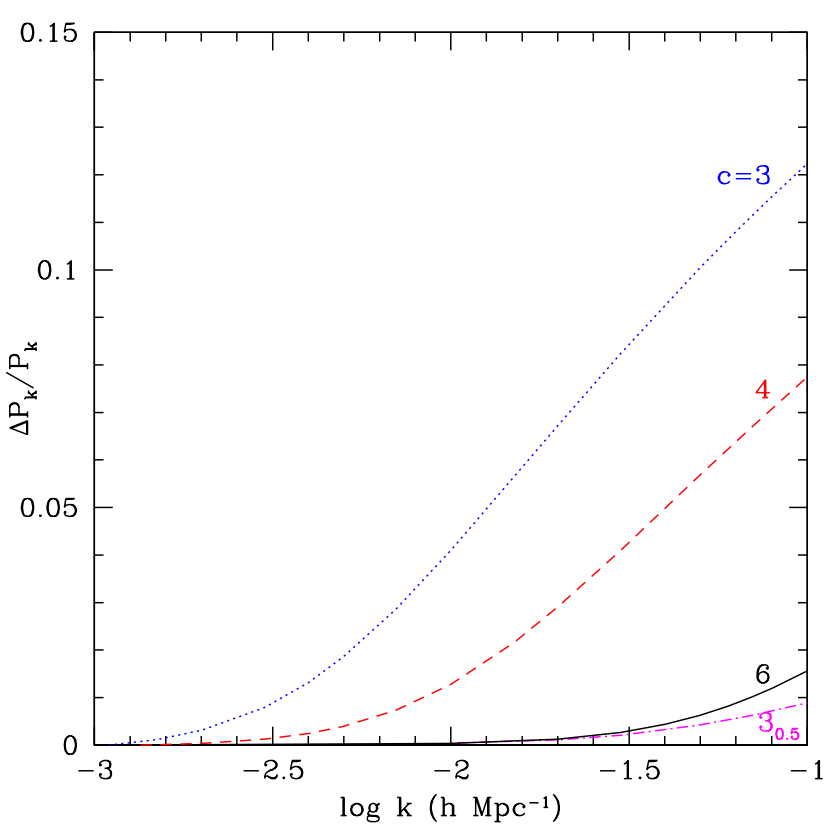

FIG. 6: The matter power spectrum in $f(R)$ theory deviates from the $\Lambda C D M$ result in a scale dependent manner. In the exponential gravity case, a wide range of values of $c$ have suppressed deviations and can be consistent with observations. The lowest curve has the same value $c=3$ as the top curve, but plots the power spectrum deviation at $a=0.5$, showing the effect of the steepness of the model.

quences, including just one more parameter than $\Lambda \mathrm{CDM}$, steep dependence that enhances solar system and structure agreements, and amelioration of fine tuning of the characteristic curvature scale. Most of the quantitative and qualitative results we present should be generally applicable to any sufficiently steep model however.

The dynamics of the effective equation of state has several interesting properties, including "sublimation" rather than thawing from a cosmological constant state. Unfortunately, even the loosened bounds on $f_{R}$ from structure constraints do not allow the equation of state to have detectable deviations from a cosmological constant expansion history - one can view the failure for exponential models, steeper than any power law, as an indication of the difficulty in distinguishing viable $f(R)$ theories' expansion from the cosmological constant. We also find an excellent fitting form for the maximum deviation from $w=-1$ in terms of the Compton scale $B$, a direct relation between the expansion and structure characteristics.

For structure growth, there exists an enlarged parameter space that gives observationally allowed deviations from $\Lambda \mathrm{CDM}$ in the matter power spectrum. In particular, because of the steep evolutionary dependence, $f_{R}$ today may be as large as $\sim 10^{-3}$ and still agree with structure constraints. The sort of model discussed here is of interest in that we have increased freedom to have a viable $f(R)$ modification of Einstein gravity that is yet distinct from standard $\Lambda \mathrm{CDM}$.

\section{Acknowledgments}

I thank Wayne Hu and Tristan Smith for useful discussions. This work has been supported in part by the Director, Office of Science, Office of High Energy Physics, of the U.S. Department of Energy under Contract No. DE-AC02-05CH11231, and World Class University grant R32-2008-000-10130-0 in Korea.
[1] R. Durrer \& R. Maartens 2008, arXiv:0811.4132

[2] R.R. Caldwell \& M. Kamionkowski 2009, Ann. Rev. Astron. Astrophys. to appear arXiv:0903.0866

[3] T.P. Sotiriou \& V. Faraoni 2008, Rev. Mod. Phys. to appear arXiv:0805.1726.

[4] Y-S. Song, W. Hu, I. Sawicki 2007, Phys. Rev. D 75, 044004 arXiv:astro-ph/0610532

[5] W. Hu \& I. Sawicki 2007, Phys. Rev. D 76, 064004 arXiv:0705.1158

[6] T. Chiba, T.L. Smith, A.L. Erickcek 2007, Phys. Rev. D 75, 124014 arXiv:astro-ph/0611867

[7] P. Zhang 2006, Phys. Rev. D 73, 123504 arXiv:astro-ph/0511218.

[8] S.A. Appleby \& R.A. Battye 2007, Phys. Lett. B 654, 7 arXiv:0705.3199.
[9] S. Tsujikawa 2008, Phys. Rev. D 77, 023507 arXiv:0709.1391.

[10] G. Cognola et al. 2008, Phys. Rev. D 77, 046009 arXiv:0712.4017.

[11] R.N. Cahn, R. de Putter, E.V. Linder 2008, JCAP 0811, 015 arXiv:0807.1346.

[12] R.R. Caldwell \& E.V. Linder 2005, Phys. Rev. Lett. 95, 141301 arXiv:astro-ph/0505494

[13] J. Khoury \& A. Weltman 2004, Phys. Rev. D 69, 044026 arXiv:astro-ph/0309411.

[14] H. Oyaizu, M. Lima, W. Hu, Phys. Rev. D 78, 123524 arXiv:0807.2462

[15] L. Pogosian \& A. Silvestri 2008, Phys. Rev. D 77, 023503 arXiv:0709.0296. 